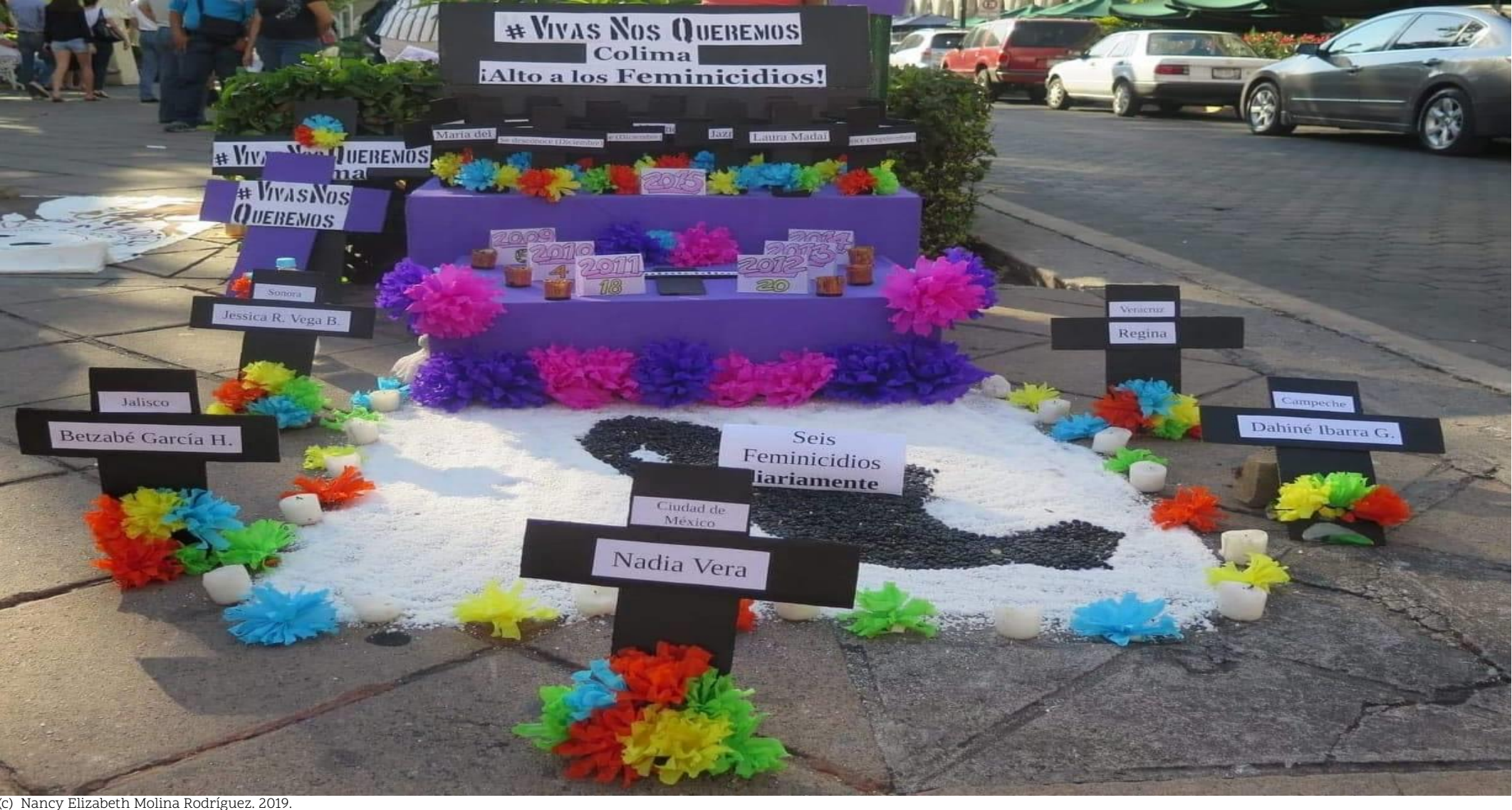

\title{
Reflexiones acerca de la implementación AVGM-Colima
}

Nancy Elizabeth Molina Rodríguez ${ }^{1}$

RESUMEN: En este documento comparto algunas experiencias acerca de mi participación como académica representante de la Universidad de Colima en el grupo de trabajo para el análisis de la solicitud de AVGM-Colima. Hago una breve descripción de las acciones realizadas por el grupo de trabajo para el análisis de la solicitud e integración del informe, después menciono algunas reflexiones desde la instalación del grupo de trabajo hasta a la implementación de la AVGM-Colima.

Palabras clave: Violencia; género; mujeres; feminicidio

\footnotetext{
${ }^{1}$ Docente de tiempo completo en la Universidad de Colima. Facultad de Psicología. Área: Humanidades y de la conducta, investigadora en la Comisión Nacional para Prevenir y Erradicar la Violencia Contra las Mujeres (CONAVIM), Universidad de Colima, contacto: molinan@ucol.mx.

UVserva No. 8 Octubre 2019 - marzo 2020, ISSN: 2448-7430.

Recepción: 04/septiembre/2019

Aceptación: 07/octubre/2019

DOI:
} 
Artículo - Derechos Humanos de las Mujeres

\begin{abstract}
In this document I share some experiences about my participation as an academic representative of the University of Colima in the working group for the analysis of the application of AVGM-Colima. I make a brief description of the actions carried out by the work group for the analysis of the request and integration of the report, then I mention some reflections from the installation of the work group to the implementation of the AVGM-Colima.
\end{abstract}

Keywords: Violence; Gender; Women; Feminicide

\title{
Introducción
}

$\mathrm{E}$

n colima el 22 de diciembre de 2014, representantes de organizaciones de la sociedad civil Fundación IUS Género, Centro de Apoyo a la Mujer (CAM) y la Comisión Estatal de Derechos Humanos solicitaron a la Secretaría Ejecutiva la declaratoria de Alerta de Violencia de Género contra las Mujeres para el estado de Colima, en adelante AVGM-Colima. El 20 de junio del 2017, la Secretaría de Gobernación (SEGOB), a través de la Comisión Nacional para Prevenir y Erradicar la Violencia contra las Mujeres (CONAVIM) emitió la Declaratoria de Alerta de Violencia de Género contra las mujeres en el Estado de Colima, en 5 municipios: Colima, Coquimatlán, Cuauhtémoc, Tecomán y Villa de Álvarez (Comisión Nacional para Prevenir y Erradicar la Violencia Contra las Mujeres, 2017). Dejando fuera el Municipio de Manzanillo en donde se han detectado casos de feminicidios, lo que genera fuertes cuestionamientos sobre cuáles son los criterios de elección de los municipios con declaratoria.

El 3 de febrero de 2015 se llevó a cabo la primera sesión del grupo de trabajo para el análisis de la solicitud de AVGM para el estado de Colima. De acuerdo con lo establecido en el artículo 36 del Reglamento de la Ley General de Acceso de las Mujeres a una Vida Libre de Violencia (LGAMVLV), el grupo de trabajo es un órgano autónomo de composición mixta, cuya función principal es estudiar y analizar la situación que guardan todos los municipios del estado de Colima señalan las organizaciones solicitantes que existe violación a los derechos humanos de las mujeres, a fin de determinar si los hechos narrados en la solicitud observan alguno de los supuestos establecidos en el artículo 24 de la Ley (LGAMVLV), según el cual, la declaratoria de alerta de violencia de género contra las mujeres, se emitirá cuando: I. Los delitos del orden común contra la vida, la libertad, la integridad y la seguridad de las 


\section{Artículo - Derechos Humanos de las Mujeres}

mujeres, perturben la paz social en un territorio determinado y la sociedad así lo reclame; II. Exista un agravio comparado que impida el ejercicio pleno de los derechos humanos de las mujeres, y III. Los organismos de derechos humanos a nivel nacional o de las entidades federativas, los organismos de la sociedad civil y/o los organismos internacionales, así lo soliciten.

Dicho grupo tiene como objetivo realizar una investigación documental y de campo a fin de identificar si en el territorio señalado por la solicitud existe un contexto de violencia que se traduce en la comisión de delitos contra la vida, la libertad, la integridad y la seguridad de las mujeres, respecto de los cuales existe impunidad o permisibilidad social y/o agravio comparado que impida el ejercicio pleno de los derechos de las mujeres.

Como primer paso el grupo de trabajo nos dimos a la tarea de analizar el documento presentado por las organizaciones solicitantes, quienes mostraron un contexto colimense que propicia la violencia contra las mujeres en sus diferentes tipos y modalidades, lo cual se ve acentuado por la falta de acceso a la justicia, lo que trae como consecuencia la impunidad.

Aquí presento algunos datos contenidos en la solicitud, por ejemplo, en el 2010 Colima ocupó el primer lugar nacional en egresos hospitalarios de mujeres por causas relacionadas a la violencia. En cuanto a las denuncias por violación sexual, en el 2005 se reportaron 27.3 violaciones por cada 100,000 mujeres. Por otra parte, la muertes violentas de mujeres señalan que el municipio de Manzanillo concentró para el 2009 el 50\% de los casos, mientras que para el municipio de Colima obtuvo el $40 \%$ del total. El medio más utilizado para cometer el asesinato, en el 2009, fueron: arma de fuego 60\%, seguido de otros métodos como el ahorcamiento, el estrangulamiento, la sofocación, el ahogamiento o la inmersión.

Además, con base al registro hemerográfico, en el periodo entre enero 2003 y noviembre 2014, se registraron un total de 101 homicidios de mujeres. Los medios por los cuales fueron asesinadas fueron: $40 \%$ arma de fuego, $18.81 \%$ arma blanca (cuchillo o machete), $14.85 \%$ por asfixia y, 9.90\% por golpes con las manos o con objetos. En tres casos las mujeres fueron ahorcadas, en dos degolladas y una niña fue quemada. Respecto al lugar de ocurrencia, el 30.69\% los cuerpos fueron encontrados en lugares donde las mujeres realizaban sus actividades cotidianas, el 39.60\% en vías de comunicación o lugares de tránsito, el 22.77\% en brechas, callejones o vías del tren y, el 6.93\% en un arroyo, cerca de un río, laguna, canal de riego o playa. En cuanto al vínculo con el homicida, en el 41.58\% la mujer tenía una relación con el homicida. De estos casos, el 59.62\% mantenía o mantuvo una relación sentimental con la víctima y el 23.80\% existía una relación de parentesco consanguíneo. Por lo anterior, y por 
Artículo - Derechos Humanos de las Mujeres

más datos recabados y plasmados en la solicitud es que se procede las investigaciones correspondientes en el marco de la AVGM-Colima.

\section{AVGM-Colima}

En la primera etapa de análisis del contexto consultamos diferentes fuentes de información como documentos, visitas a instituciones, entrevistas a personal de instituciones, representantes de organizaciones de las sociedad civil y víctimas. En esta fase de recolección de datos con las instituciones, se observó cómo en las instituciones de gobierno responsables de prevenir, atender y sancionar la violencia contra las mujeres, el personal encargado de proporcionar servicios, en su mayoría, carecían de una capacitación en derechos humanos y perspectiva de género y desconocían protocolos de atención, así como la NOM-046. Por otro lado, se observó la deficiencia y la falta de recursos humanos y materiales suficientes para solventar las demandas de las mujeres víctimas de violencia.

Al reunirnos con las personas representantes de organizaciones civiles y a las mujeres víctimas de violencia, se identificó que los procesos de atención a las mujeres son obstaculizados por el mismo personal que las atiende, desanimando a poner la denuncia o impidiendo trámites como órdenes de protección, por mencionar algunos, lo que agrava la situación de vulnerabilidad de las mujeres en Colima.

Cuando tocó el turno de visitar a las autoridades responsables de las instituciones de prevenir, tratar y sancionar la violencia, se les hizo saber las problemáticas encontradas en las fuentes de consulta anteriores, la respuesta ello fue que, los secretarios del gabinete estatal se comprometieron a tomar acciones para remediar la situación y llevar a cabo las observaciones que se hicieron en el informe.

En la segunda fase para integrar el informe de la AVGM-Colima, tal como lo marca la LGAMVLV, se realizó el análisis de los datos obtenidos de: la solicitud, la información de gobierno; visitas in situ a instalaciones; entrevistas a organizaciones de la sociedad civil, a las solicitantes, a mujeres víctimas de violencia de género y/o familiares, autoridades encargadas de la atención a mujeres víctimas de violencia y procuración de justicia, organismos autónomos de derechos humanos, expertos en el tema; así como información oficial, de los medios de comunicación, y de estudios académicos. 


\section{Artículo - Derechos Humanos de las Mujeres}

La dinámica para la integración del informe consistió en enviar por correo los análisis de los indicadores que nos fueron asignados previamente, para luego discutirlas con el resto del grupo de manera presencial en las instalaciones de la CONAVIM. Fue una de las jornadas más largas, eran demasiadas observaciones que se le hicieron al Estado, y tuvimos que decidir por incluir solo diez de acuerdo al formato preestablecido para el informe por la CONAVIM. El debate entre el equipo de trabajo fue arduo, dado que las perspectivas académicas y las institucionales son diferentes, lo cual enriquece el análisis, pero, no hay equilibrio en las votaciones debido a que el equipo institucional es mayoría, esto se podría solventar incluyendo_a personas de la sociedad civil expertas en el tema que pertenecen a colegios de profesionales, aunado a tomar decisiones basadas en evidencias y en criterios de valoración de los riesgos para la vida de las mujeres, el ciclo de la violencia y los factores que la incrementan.

Cabe aclarar que quienes conformamos la parte académica, después de analizar el contexto de sistemática vulneración de los derechos humanos de las mujeres, siempre nos quedó claro que era indiscutible declarar la alerta de violencia de género hacia las mujeres. Sin embargo, una sección de la parte institucional insistía en que de alguna manera se considerará no declararla, llegando incluso a solicitar aplazar el tiempo para la entrega de más evidencias, lo cual así fue, pues se le otorgó seis meses más. En adelante estos aplazamientos fueron ya costumbre, tanto que hasta la fecha de este escrito no hemos tenido reunión para decidir si se continúa o se detiene la AVGM-Colima.

En cuanto a las medidas que deberá tomar el Estado así como a la evaluación de éstas, desde mi punto de vista, deberán considerar que se está interviniendo con una sociedad compleja donde por un lado, a corto a corto plazo se requieren acciones inmediatas de protección a la integridad y la vida de las mujeres y las niñas, en tanto que como dice Segato (2016), se modifica la imaginación de género que tiene tiempos muy largos. Para estas medidas de modificación de procesos psicológicos, sociales y prácticas culturales, dejar metas a mediano y largo plazo, así como su correspondiente evaluación realizada por un grupo de expertos que den continuidad al proceso, independientemente de quien esté a cargo en las instituciones involucradas.

Desde la integración del informe hasta la evaluación de la implementación se requiere que existan criterios cualitativos y cuantitativos para que el grupo de trabajo tome las decisiones y emita las conclusiones de manera sistematizada y rigurosa, y con ello crear un sistema de seguimiento con validez y confiabilidad metodológica.

UVserva No. 8 Octubre 2019 - marzo 2020, ISSN: 2448-7430. 


\section{Artículo - Derechos Humanos de las Mujeres}

Se debe cumplir cabalmente con la periodicidad en que se programan las reuniones y el seguimiento del trabajo realizado, para ello es necesario considerar los eventos políticos como las campañas electorales que restringe mucho el actuar del Estado, así como que la CONAVIM cuente con el suficiente personal para dar cobertura a todas las alertas vigentes.

Continuar con la integración del sector académico en el grupo de análisis de la AVGMColima, porque aportan la perspectiva metodológica y teórica de la disciplina en la cual son expertos/as, así como integrar dichas aportaciones a los informes que se emiten. En este mismo sentido, incluir en el grupo de trabajo a personas de la sociedad civil expertas en el tema, que pertenecen a colegios de profesionales, puesto que tienen de primera mano la experiencia de su ejercicio profesional con las mujeres víctimas de violencia, la idea es la articulación de los esfuerzos entre el Estado, la academia y la sociedad civil.

En cuanto a la implementación AVGM-Colima, el proceso ha evidenciado, en repetidas ocasiones, cómo los tres niveles de gobierno carecen de sensibilidad y compromiso político ante el problema de la violencia de género hacia las mujeres y niñas, hecho que se observó en la falta de recursos materiales y personal capacitado en el enfoque de derechos humanos y perspectiva de género, y en el aplazamiento y no programación de recursos, planes y programas que deriven en acciones que protejan la vida de las mujeres. La propuesta ante estos deficiencias del sistema, es modificar de raíz las creencias entorno a como afirma Segato (2016) considerar a las mujeres y sus vidas como "menores" y a relegar sus temas al ámbito de lo íntimo, de lo privado, a verlo como tema de minorías derivando en ver la violencia de género como problema minoritario.

A la par que ha permitido visibilizar la violencia de género que viven las mujeres y las niñas colimenses, al poner en la mesa de discusión el problema de la violencia y discriminación por género en la agenda política del gobierno del Estado, lo que debe de tener como consecuencia mínima sentar las bases para generar una estructura política, administrativa y legal sobre la cual deberá trabajarse en los siguientes años con acciones muy puntuales, y a contratar gente comprometida en velar por la integridad y vida de las mujeres.

La AVGM-Colima ha contribuido a sistematizar en los informes emitidos por el Estado, los delitos en contra de las mujeres, la violación a sus derechos, las deficiencias en los sistemas de prevención, atención, tratamiento e impartición de justicia, así como las acciones que se están implementando. 


\section{Artículo - Derechos Humanos de las Mujeres}

También, ha impactado de manera indirecta en el papel de las organizaciones civiles quienes de manera extraoficial dan seguimiento a la implementación de las acciones, y ejercen presión al Estado mediante la realización de comunicados de prensa, manifestaciones públicas, mesas de debate, campañas en redes sociales y plantones, para mostrar que la violencia de género en contra de las mujeres ha aumentado en lugar de disminuido, contrario a lo que promueve la AVGM-Colima.

Si algo confirmo con la experiencia de participar en la AVGM-Colima es que, no es por el hecho de instalar la declaratoria como se eliminará la violencia de género contra las mujeres, la mente de los hombres misóginos y feminicidas no dejará de considerar a las mujeres como objeto de su propiedad por el hecho de que se ha declarado la AVGM-Colima, porque los hombres feminicidas, muchas veces, no se enteran de que existe una ley que castiga la violencia contra ellas, y tampoco con estar informados es suficiente para el cambio. En otros casos, puede que los hombres se enteren de que es un delito, sin embargo, la ejercen porque saben que no recibirá castigo debido a que no hay quien lo haga, o porque si hay quien corrija, pero, éste no lo hará por no considerarlo importante, por complicidad, por no meterse en problemas "privados", o por soborno. Esto genera la idea en los hombres violentos que la violencia es mal vista, pero no castigada. Aunque, coincido con Segato (2003:6), en que "apostar simplemente a la criminalización y a el encarcelamiento, sobre todo si éste no es acompañado por procesos reeducativos, significa investir en la misma lógica de que se alimenta la violencia".

En los casos en que, si existe el castigo, éste con frecuencia no suele ir acompañado de una ayuda psicológica y social con perspectiva de género que permita una reflexión crítica y constante sobre el ejercicio de su violencia y la construcción de su masculinidad. Este punto me parece muy importante dado que como afirma Deschner (1984), si no existe un factor que irrumpa con este ciclo de violencia, este aumentará su frecuencia y gravedad, como ya lo estamos viviendo. Por lo anterior, estoy de acuerdo con lo que afirma Rita Segato (2003:4):

No es por decreto, infelizmente, que se puede deponer el universo de las fantasías culturalmente promovidas que finalmente conducen al resultado perverso de la violencia, ni es por decreto que podemos transformar las formas de desear y de alcanzar satisfacción constitutiva de un determinado orden sociocultural, aunque al final se revelen engañosas para muchos. Aquí el trabajo de la conciencia es lento pero indispensable.

Por otra parte debe de considerarse que desde el momento mismo en que se pide la AGVMColima, las solicitantes han registrado ya un contexto cultural y social que reproduce sistemáticamente la violencia contra las mujeres, es decir, ya hay un proceso avanzado de

UVserva No. 8 Octubre 2019 - marzo 2020, ISSN: 2448-7430. 
Artículo - Derechos Humanos de las Mujeres

naturalización de la violencia, y en nuestro caso se encuentra estrechamente relacionado con prácticas culturales de corrupción, impunidad y simulación en aras de preservar el statu quo del Estado, quien además, ha perdido la capacidad para enfrentar al crimen organizado que domina a nuestra sociedad y nos vulnerabiliza aún más a las mujeres.

También, reflexiono que las expectativas de la AVGM-Colima, son muy altas y los alcances inmediatos, hasta ahora, muy bajos, puesto que, al ser un mecanismo de emergencia destinado a generar respuestas inmediatas de las autoridades responsables, cuando se presentan situaciones graves de violencia, no ha cumplido con esta función pues siguen permeando las ideas conservadoras en quienes tienen el poder de tomar decisiones para acatar las recomendaciones. Por ejemplo, los/as legisladores/as no ha logrado tipificar el feminicidio que no cuentan con un tipo penal adecuado, por contener elementos adicionales innecesarios o desarrollan las razones de género con elementos subjetivos que dificultan o impiden la acreditación del delito (Aguascalientes, Baja California, Baja California Sur, Chihuahua, Durango, Guanajuato, Michoacán, Nayarit, Puebla, Quintana Roo, Tabasco, Tlaxcala, Yucatán y Chihuahua). Situación similar es el caso de Jalisco en el que se recomendó derogar los delitos de adulterio y abuso sexual infantil que sancionan la misma conducta que el delito de violación, pero con penas menores, esto tampoco ha sido modificado (Observatorio Ciudadano Nacional del Feminicidio, 2018).

No cabe duda que, todas queremos que no sucedan más feminicidios, pero, el problema implica que "los feminicidios no suceden solos" es decir, alguien comete feminicidio, ese alguien mayoritariamente es un hombre, que como ya lo han dicho las teorías feministas y la misma LGAMVLV, es un problema del sistema patriarcal que produce la división de los géneros femenino y masculino, donde el masculino cree que tiene poder y dominio sobre el femenino, por eso, las acciones deben de estar dirigidas a trabajar no solo con las víctimas, quienes por su puesto son muy importantes, además es necesario trabajar para cambiar los patrones de masculinidad que fomentan la apropiación del cuerpo y de la vida de las mujeres y en estrategias con mayor énfasis en el ámbito comunitario y social en búsqueda de tejer lazos solidarios y respetuosos entre los géneros, e instalar prácticas institucionales éticas apegadas a respetar los derechos humanos de las mujeres.

Al respecto el gobierno del estado de Colima, en el 2016 a través del Instituto Colimense de las Mujeres (ICM) implementó la campaña "Vivir sin violencia es posible, donde los hombres y los niños son parte de la solución", basada en la propuesta de ONU Mujeres HeForShe, se repartieron materiales prácticos con el eslogan, se realizó promoción ambiental, en medios 


\section{Artículo - Derechos Humanos de las Mujeres}

electrónicos y digitales. A la par, a través del Consejo Estatal para la Prevención y Atención a la Violencia Intramiliar (CEPAVI) ha diseñado un programa de atención especializada para hombres generadores de violencia de género, sin embargo, este se encuentra solo en el municipio de Colima. Como actividad adicional de prevención se brindó la conferencia magistral denominada "La importancia del programa de atención de hombres generadores de violencia familiar y de género en la eliminación de la violencia contra las mujeres" impartida por el Mtro. Roberto Garda Salas, director de Hombres por la Equidad A.C. en el marco del Día Internacional de la Eliminación de la Violencia contra a las Mujeres (Instituto Colimense de las Mujeres, 2018).

Aunado a lo anterior, se estableció capacitar a servidoras y servidores públicos para la atención a hombres generadores de violencia $y$, brindar atención especializada para hombres generadores de violencia de género, basado en la perspectiva de género y con enfoque de derechos humanos. Sin embargo, hasta la fecha no se ha reportado datos de su implementación. Se requiere activar la red interinstitucional que el mismo CEPAVI tiene para capacitar a servidores públicos y así poder brindar atención especializada a los hombres y prevenir la violencia. Así mismo, y con el fin de formar a futuros profesionales, se puede incluir en esta red a instituciones formadoras de profesionistas como las universidades, para que sus estudiantes se formen en estos temas, considerando áreas como la medicina, psicología, trabajo social, derecho y criminología.

Finalmente, cabe mencionar lo que afirma Domínguez (2015:74), en México "simular es gobernar", existe una práctica generalizada entre la población mexicana de que la política es una mascarada, una escenificación ritual de las ideologías. Dos son los mecanismos a través de los cuales se reproduce esta práctica: por un lado, en la política se valoran los lazos de la complicidad, las relaciones mafiosas, el nepotismo, el clientelismo y el intercambio de favores. Por otro, la ilegalidad como forma de vida a través de prácticas gubernamentales fraudulentas y los métodos coercitivos de formación de consensos sociales como la censura y el corporativismo, como norma que rige los procesos económicos y políticos. Lo que tiene como consecuencia hechos criminales generalizados y continuos e impunes que define una forma de gobierno.

Por lo tanto, se requiere un cambio radical a nivel de estructuras políticas, económicas y culturales, es decir, en la forma en cómo está concebido el Estado mexicano en todos sus niveles, cuyas prácticas de impunidad, corrupción y simulación contribuyen al mantenimiento y reproducción de todas las formas de discriminación y violencia contra las mujeres. 
Artículo - Derechos Humanos de las Mujeres

\section{Referencias}

Comisión Nacional para Prevenir y Erradicar la Violencia Contra las Mujeres (2015) Informe del Grupo de Trabajo conformado para atender la solicitud de Alerta de Violencia de Género contra las Mujeres en el estado de Colima. En Secretaría de Gobernación. Consultado el 3 de mayo 2019. Recuperado de https://www.gob.mx/conavim/documentos/solicitud-de-alerta-de-violencia-degenero-contra-las-mujeres-en-el-estado-de-colima

Comisión Nacional para Prevenir y Erradicar la Violencia Contra las Mujeres (2017) Declaratoria de Alerta de Violencia de Género contra las Mujeres en el estado de Colima. En Secretaría de Gobernación. Consultado el 3 de mayo 2019. Recuperado de https://www.gob.mx/conavim/documentos/solicitud-de-alerta-de-violencia-degenero-contra-las-mujeres-en-el-estado-de-colima

Deschner, J. P. (1984) How to end the hitting habit. New York: Free Press.

Dominguez, H. (2015) Nación Criminal, narrativas del crimen organizado y el estado mexicano. México: Ariel.

Instituto Colimense de las Mujeres (2018) Informe Parcial de Cumplimiento de la Alerta de Violencia de Género contra las Mujeres para el Estado de Colima. Manuscrito no publicado.

Ley General de Acceso de las Mujeres a una Vida Libre de Violencia. Diario Oficial de la Federación. México 13 de abril de 2018.

Observatorio Ciudadano Nacional del Feminicidio (2018) Informe implementación del tipo penal de feminicidio en México: desafíos para acreditar las razones de género 2014 2017. En Observatorio Ciudadano Nacional del Feminicidio. Consultado el 5 de octubre 2019. Recuperado de

https://observatoriofeminicidio.files.wordpress.com/2018/05/enviando-informeimplementaciocc81n-del-tipo-penal-de-feminicidio-en-mecc81xico-2014-2017-1.pdf

Segato, R. L. (2003) Las estructuras elementales de la violencia: contrato y status en la etiología de la violencia. Conferencia presentada en la abertura del Curso de verano sobre Violencia de Género, Madrid, España.

Segato, R. L. (2016) La guerra contra las mujeres. Madrid: Traficante de sueños. 